\title{
Estimation of the Solar Power Tower Heliostat Position using Neural Network
}

\author{
A.Zeghoudi \\ URMER Research unit, Tlemcen University, \\ B.P. 119, Tlemcen, Alegria
}

\author{
A. Chermitti \\ URMER Research unit, Tlemcen University, \\ B.P. 119, Tlemcen, Algeria
}

\begin{abstract}
The alignment and control of heliostats have been one of most issues in solar tower power. In this paper, we review our work to estimate the alignment of solar tower heliostat field based on the combination of two control systems (open loop and closed loop system) using a neural network approach. Several factors influence the path of each heliostat such as the azimuth, the elevation time, the date, the position of the sun, the plant location, the height of the tower and the heliostat size and slope. Firstly, we have modeled the heliostat position using astronomical formulas. Secondly, the neural network model is trained using data from January of three heliostats for both paths. Then, it was tested with another heliostat position for the month of March. The accuracy of the model was evaluated using the mean absolute error (MAE) and absolute percentage error (MAPE). The simulation results show the accuracy of the proposed for estimating the heliostat position without detecting the position of the sun.
\end{abstract}

\section{Keywords}

Solar tower power, heliostat field, open loop and closed loop system, neural networks.

\section{INTRODUCTION}

The heliostat field is the major cost component of the thermal solar tower power plant and the optical quality of the heliostats has a significant impact on the field efficiency and affect the performance of the power plant. It is therefore a permanent goal to decrease their manufacturing cost. The heliostat fields are used to reflect and concentrate the sunlight toward the receiver target in the upper part of the tower. The concentrated radiation is absorbed then it transformed into a thermal energy for producing electricity. The heliostat is a key optical device which tracks the sun and concentrates the solar radiation onto the immoveable target. Generally, the heliostat contributes about $50 \%$ [1] to the total cost of system and its annual energy loss is about $47 \%$ [2]. The optical efficiency of field strongly affects the generation efficiency of the plant. Therefore, the design of the heliostat field layout is important. There are considerable existing procedures for designing heliostat field such as HELIOS [3], ASPOC [4], HFLCAL [5],
RCELL [6], DELSOL [7] etc. These procedures were all written in the eighties of the last century and existed disadvantages, For examples, the calculated results are not accurate, they are difficult to use and lack graphic output. Recently [8].

The performance of solar tower power depends strongly on the solar field efficiency which is related to the heliostat design, the field layout, the tracking system and control system. In this section, the published studies focused on the heliostat field are reviewed and their results are briefly reported. Also, methods and techniques used or proposed for enhancing the heliostat and the heliostats field performance are sketched out.

In this work, a new method of modeling alignment field of heliostats is developed by employing the MATLAB software. The rest of this paper is organized as follow, section 1 gives a background about the parameter influences on the solar transmission process. In addition, we present the different type of the heliostat sun tracking. Section 2 introduces the proposed method of alignment of the heliostat solar tower. Section 3 shows the estimation method using the neuron network, Section 4, shows the simulation results of the modelling and estimation of the heliostats and the final section is devoted to the conclusion.

\section{PARAMETER INFLUENCES ON SOLAR TRANSMISSION PROCESS AND CONTROL SYSTEM}

According to the solar radiation model, the solar altitude and azimuth angles change its value in time, which will have direct influence on the optical efficiency of the heliostat field and heat flux distribution of the absorber [9]. The volumetric receiver is mounted on a receiver tower to collect the solar rays concentrated by the heliostat field. So the variation of receiver mounting height will result in a great change of heliostat field's light path and further effects the optical efficiency of the heliostat field and flux distribution of the absorber. 
Due to wind, self-weight and mechanical error of heliostats, the concentrated solar rays will deviate from their preselected path during the operation of heliostats, which further influences the final heat flux distribution in the absorber. As the tracking error increases, the light spot range on the heliostat field's focal plane is enlarged and the interception loss increases. Thus, there will be less solar rays entering into the volumetric receiver and absorbed by the absorber.

As showed in Figure 1, the annual mean field efficiency and maximum heat flux of the absorber exhibit a decrease trend as well. When the tracking error increases from 0 to $6 \mathrm{mrad}$, the annual mean field efficiency decreases from $67.03 \%$ to $61.30 \%$ and though the absorbed energy still concentrates at the top area of the absorber, [9].

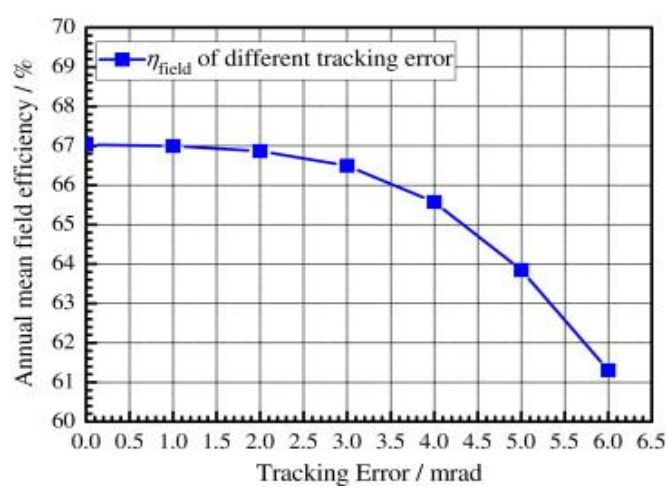

\section{Fig 1: Annual mean field efficiency of different tracking} error.

In the solar field, each heliostat tracks the sun to minimize the cosine effect, and therefore maximize the solar energy collection through positioning its surface normal to the bisection of the angle subtended by Sun and the solar receiver. Heliostat sun tracking can be classified either as an open loop system or as closed loop system [10]. The open loop system is based on astronomic formulae relating the sun's position to the system geometry. This system is reliable-low cost and it is recommended for larger solar field because the heliostat is under computer control. On the other hand, the closed loop system uses a sensor to track the sun. This system is more accurate and very useful for small heliostat fields. However, this system suffers from lower performance during cloudy period. Two sun-tracking methods are usually applied in CRS, i.e., the Azimuth-Elevation (A-E) and Spinning-Elevation (S-E) [11]. Compared with A-E the S-E tracking method allows more solar energy collection at the receiver and reduces spillage losses by $10-30 \%$ [11].

Chen et al. [12] have analyzed the optical performance of two different sun tracking methods at the level of a single heliostat and at that of a heliostat field. They have been considered the case of a fixed geometry non-imaging focusing heliostat using Spinning-Elevation (S-E) axis and the case of a spherical geometry heliostat using Azimuth-Elevation (A-E) axis are considered. They have found that the S-E tracking system can reduce the receiver spillage losses by $10-30 \%$. Moreover, the S-E tracking provides much more uniform concentrated sunlight at the receiver without huge variations with the time of day compared to the A-E system.

\section{MODELING}

The main objective of our modeling is to determine the position of the sun relative to a fixed point on the earth, from an algorithm that allows us to find the azimuth angle and elevation of the sun a leave. And identify the path of the normal of a heliostat solar power tower changes during a day. By the open-loop system that is based on astronomical formulas for the position of the sun to the geometry of the system.

\subsection{The heliostat movements}

We determine the two angles " $\alpha$ " and " $\gamma$ " of each heliostat for the solar tower power plant according to the position (distance tour heliostat), the date, time and latitude of the site. Assuming that the height of the tower, the size of the aperture of the receiver, the size and number of heliostats are fixed, then the layout of the fields is determined by six parameters such as the slope, the distance right-left between adjacent heliostats, the distance back and forth between adjacent rows, the distance between the tower and heliostat and two projection (North-South, East-West). These parameters are fixed and allowed for varied within the programming; the method allows a layout and calculates the two movements (azimuth, elevation) of each heliostat in a solar tower. The flowchart of the procedure is shown in Figure 2.

\begin{tabular}{|c|c|c|c|c|}
\hline $\begin{array}{l}\text { select } \\
\text { the site }\end{array}$ & $\begin{array}{l}\text { calculate the } \\
\text { position of } \\
\text { the sun }\end{array}$ & $\begin{array}{c}\text { imput } \\
\text { paramétres }\end{array}$ & $\begin{array}{c}\text { Locate } \\
\text { heliostats }\end{array}$ & $\begin{array}{l}\text { calculate the } \\
\text { azimuth and } \\
\text { elevation }\end{array}$ \\
\hline
\end{tabular}

Fig 2. The flow chart of the procedure.

A case study is conducted on 9 heliostats positioned following:

- the target angular height (solar receiver) $15^{\circ}, 30^{\circ}$ and 45 relative to the center of the mirror.

- The azimuths of the target relative to the center of the mirror is from $-45^{\circ}, 0^{\circ}$ and $45^{\circ}$.

These configurations correspond to investments of heliostats as they appear in Figure 3, where the height of the tower is 100 meters. 


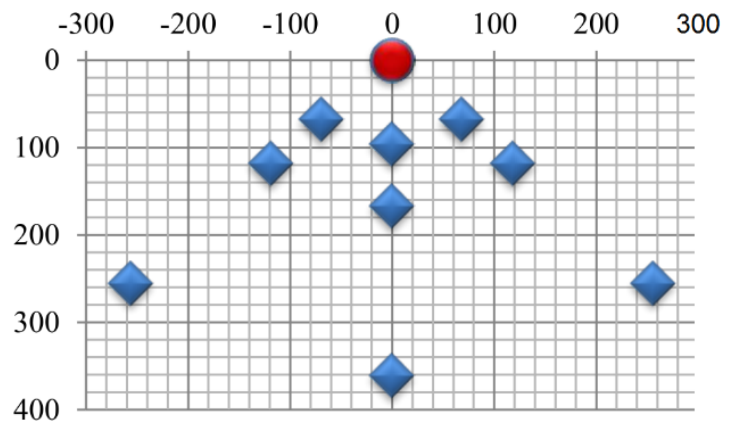

Fig 3. Placement of heliostats (m) depending on the height of the tower.

\subsection{Estimation neural network}

This example illustrates the estimation of the alignment field of heliostats with a forecasting system in Matlab, a nonlinear regression model (neural network) to predict the movements of the heliostat field of a solar tower power plant; the model is trained on data calculated by the first procedure (Figure 4) of January.

\subsubsection{Building the model and forecasting:}

The three steps to building the forecaster include creating a matrix of predictors from the historical data, selecting and calibrating the chosen model and then running the model.

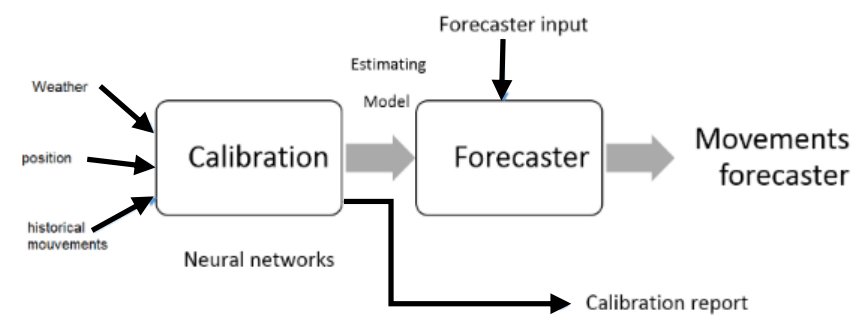

Fig 4. Estimation model for alignment of heliostat.

\subsubsection{Learning algorithm}

The neural network is trained using Levenberg-Marquardt back propagation algorithm. It is a network training function that updates weight and bias values according to LevenbergMarquardt optimization. It is often the fastest backpropagation algorithm for training moderate-sized feedforward neural networks (up to several hundred weights), although it does require more memory than other algorithms [13].

Like the quasi-Newton methods, the Levenberg-Marquardt algorithm was designed to approach second-order training speed without having to compute the Hessian matrix. When the performance function has the form of a sum of squares (as is typical in training feedforward networks), then the Hessian matrix can be approximated as

$$
H=J^{T} . J
$$

and the gradient can be computed as

$$
g=J^{T} e
$$

where $\mathrm{J}$ is the Jacobian matrix that contains first derivatives of the network errors with respect to the weights and biases, and $\mathrm{e}$ is a vector of network errors. The Jacobian matrix can be computed through a standard backpropagation technique that is much less complex than computing the Hessian matrix.

The Levenberg-Marquardt algorithm uses this approximation to the Hessian matrix in the following Newton-like update [14]:

$$
x_{K+1}=x_{k}-\left[J^{T} J+\mu I\right]^{-1} J^{T} e
$$

When the scalar $\mu$ is zero, this is just Newton's method, using the approximate Hessian matrix. When $\mu$ is large, this becomes gradient descent with a small step size. Newton's method is faster and more accurate near an error minimum, so the aim is to shift toward Newton's method as quickly as possible. Thus, $\mu$ is decreased after each successful step (reduction in performance function) and is increased only when a tentative step would increase the performance function. In this way, the performance function is always reduced at each iteration of the algorithm.

\section{Selection of neural network:}

Framing of neural network development.

a. Number of input variable $=5$

b. Number of output $=1$

c. Number of input layer neurons $=5$

d. Number of Hidden layer neurons $=20$

e. Number of Hidden layer $=1$

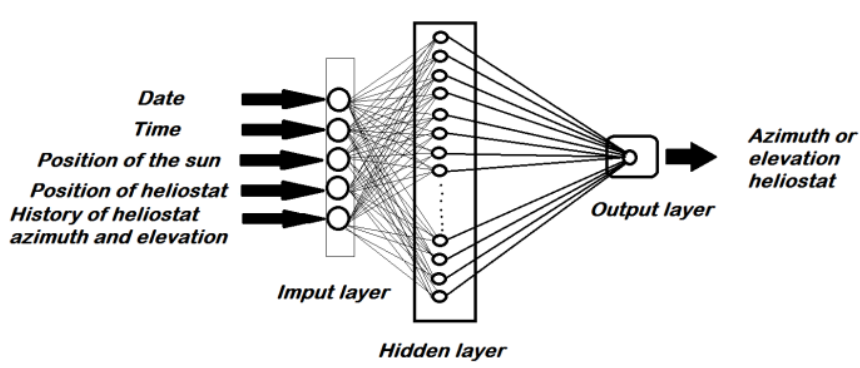

Fig 5: Neural Network movement estimating model

\section{$\underline{\text { Selection of training parameter }}$}

a. Number of epochs $=31$ and 55(number of iterations required to reach to the final goal).

b. Error tolerance $=2.221 \mathrm{e}-07$ and $4.4023 \mathrm{e}-08$ (for the accuracy ) 


\section{EXPERIMENTAL RESULTS}

\subsection{Number of Hidden Neurons and Delays}

For a small mean square error MSE, we have increased in this paper the number of neurons in the hidden layer and the number of delays in the tapped delay lines. We found the use of 20 neurons in hidden layer with 55 delay ( in the case of elevation ) and 31 delay ( in the case of azimuth ) give the best results.

\subsection{Mean Squared Error}

The mean squared error is the mean squared normalized error performance function. The error is the difference between the output and the target. Validation vectors are used to stop training early if the network performance on the validation vectors fails to improve or substantially remains the same [13]; the best validation performance is $2.221 \mathrm{e}-07$ at epoch 31. As showed in Figure 6. It shows that the errors of training, validation and testing decreased.

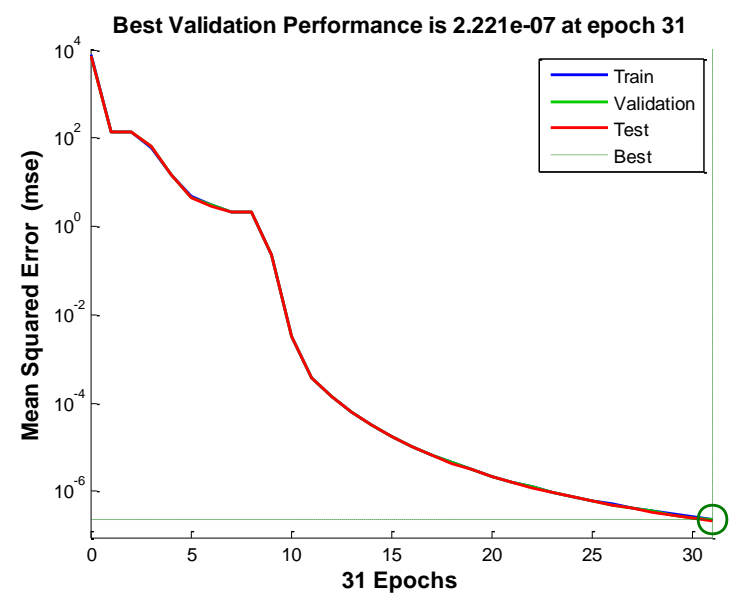

Fig. 6. The best validation performance is $2.221 \mathrm{e}-07$ at epoch 31 .

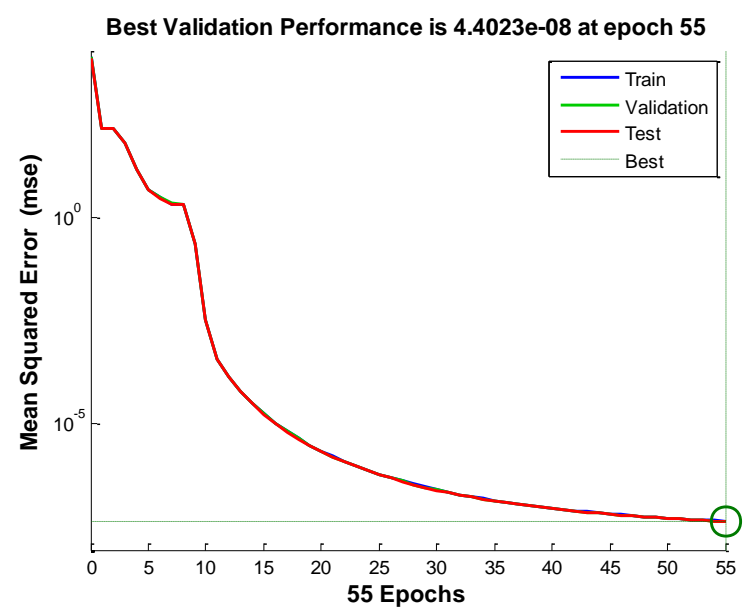

Fig. 7. The best validation performance is $4.4023 \mathrm{e}-08$ at epoch 55.

\section{RESULTS AND DISCUSSION}
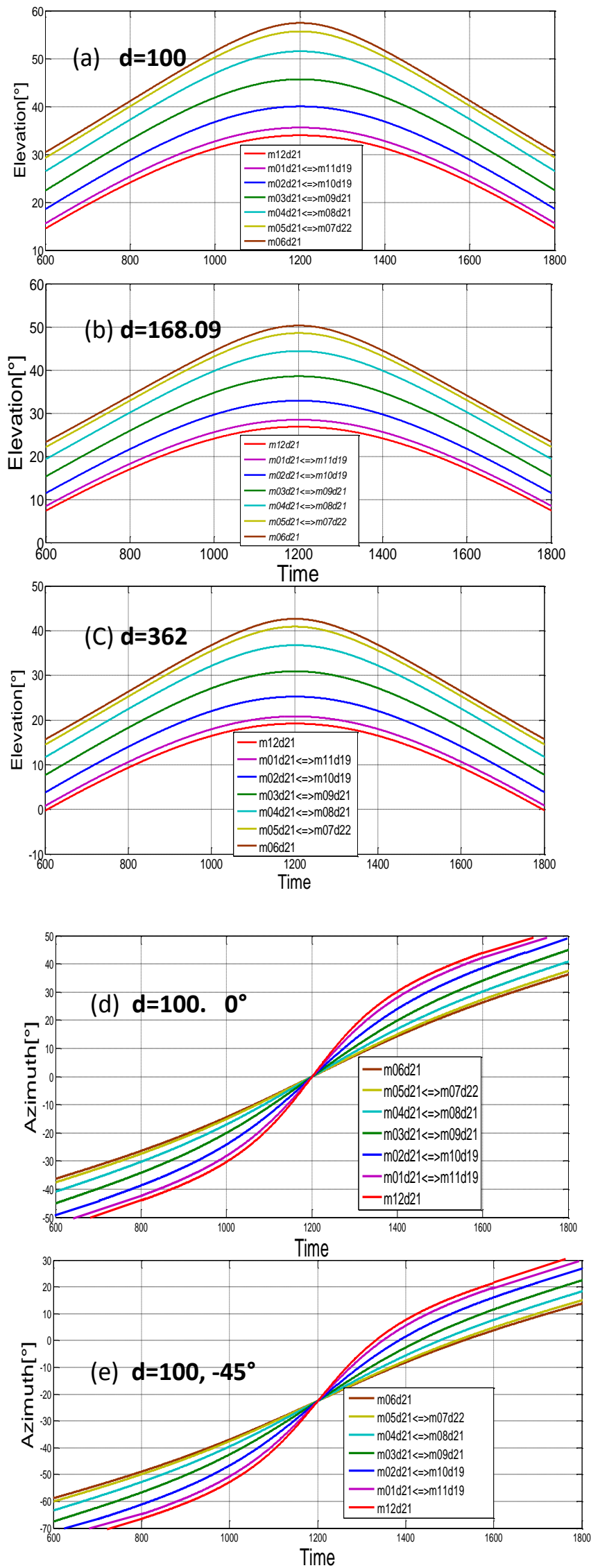


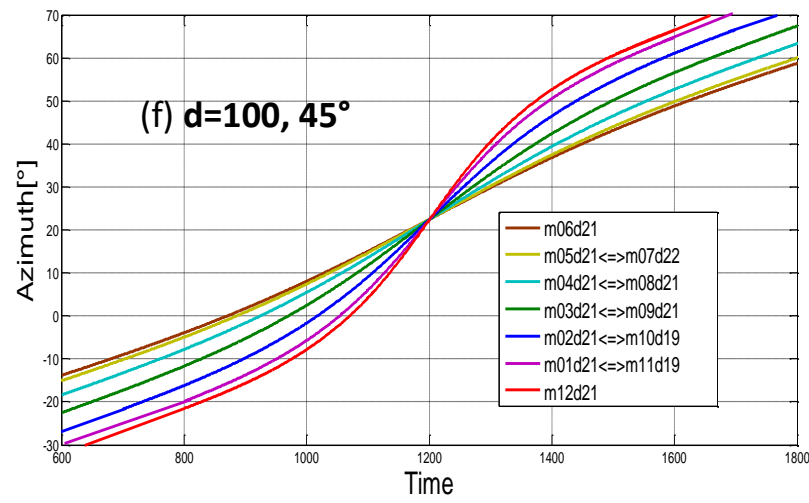

Fig 8. Elevation tracking angle, a,b,c and azimuth tracking angle, d,e,f for the 6 heliostats for the whole year.

The graphs of Figures $\mathrm{a}, \mathrm{b}, \mathrm{c}, \mathrm{d}$, e and $\mathrm{f}$ represent the path of the $\mathrm{H}$ point in the sky, in the northern hemisphere, the Sun races are symmetrical to the south. The tower is always located to the south and heliostats to its north.

For a heliostat located in front of his target, the trajectories are symmetric with respect to the south as shown in Figures. a, b and $\mathrm{c}$ for elevation and figure $\mathrm{d}$ for azimuth, the heliostat rises early in the day, reached its highest point at 12 hours and decreasing until the end of the day, This is the general appearance of the races of the Sun. The races are the highest to the summer solstice and lower towards the winter.

Amplitudes race elevation is even more important than the target height is large, that is to say, especially since the heliostat is close to the tower.

The deviation in $\pm 45^{\circ}$ of heliostats with respect to the turnsouth axis causes a change of the heliostat races (Figure e and Figure f). The angle $0{ }^{\circ}$ points are not located around 12 hours length races is greater in summer than in winter, heliostats work longer in summer than in winter too, the same variation of amplitude in azimuth and elevation is higher in summer than in winter, the heliostat consumes more.

The relative position of a heliostat relative to the tower plays an important role in the consumption of a heliostat for racing axes vary greatly. The tower itself can be a barrier to the reflection of the light flux. Its height should not be too large to avoid a heliostat alignment between Sun and turn. The courses are valid for a mirror whose center of gravity lies on the axis of rotation of the azimuth or elevation.

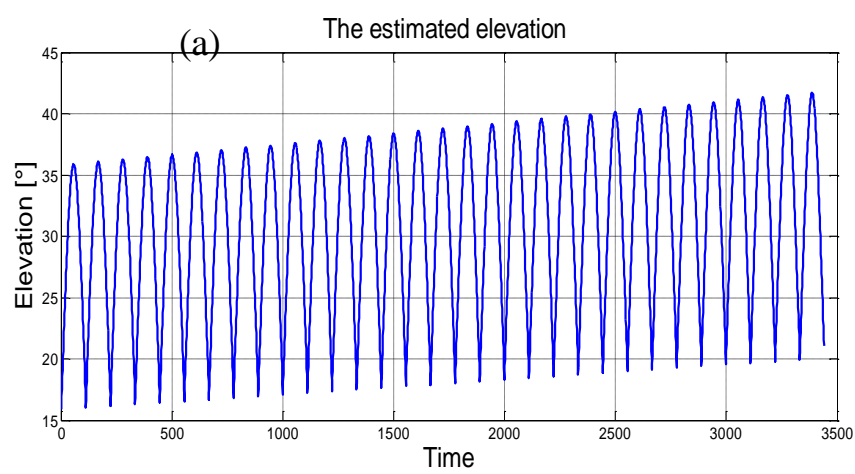

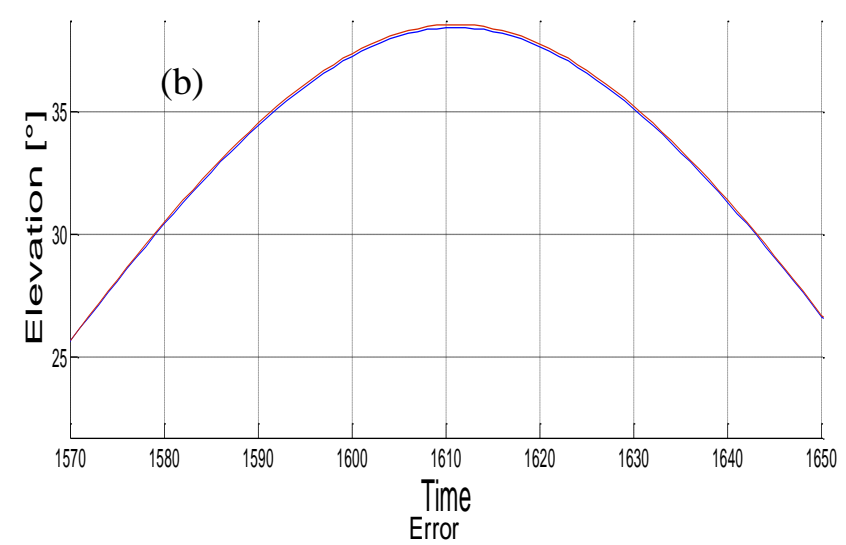

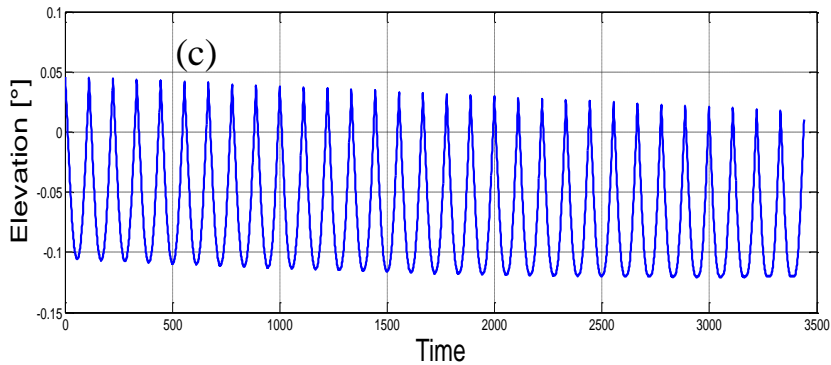

Fig .9 Comparison between the modeled results and estimated by neural network of heliostat elevation.
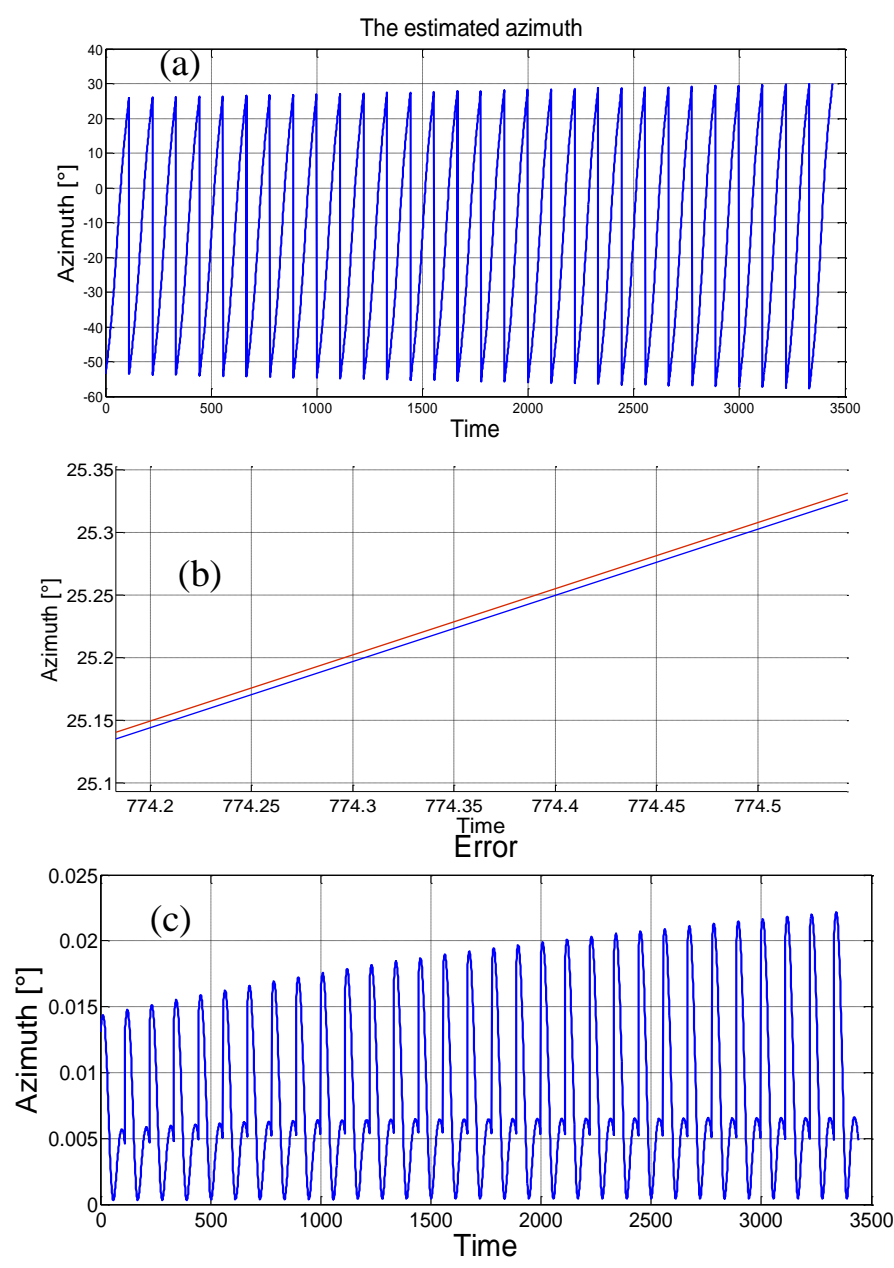

Fig 10. Comparison between the modeled results and estimated by neural network of heliostat azimuth. 


\subsection{The estimation results by neural network}

The neural network model is trained on data from January 3 matrices consisting of elevation (angle of $15,30^{\circ}$ and $45^{\circ}$ ) and 3 different heliostat azimuth $\left(45^{\circ}, 0^{\circ}\right.$ and $\left.-45^{\circ}\right)$ and tested in March with other heliostat position $(\mathrm{d}=150$, angle $=26.56$ ).

The accuracy of the model on the off sample is calculated using the mean absolute error (MAE) and absolute percentage error (MAPE)

The two paths azimuth and elevation are superposed in Figure 9. (a) and figure 10. (a). The results are shown in detail (Figures. b), where it is seen that the curves according to the deviations are less than 0.0702 degrees in elevation and azimuth to 0.0081. Figure 9 and 10 (c).

The mean absolute error (MAE) and the absolute percentage error (MAPE) are presented in the following table.

Table 1. Result of NN model.

\begin{tabular}{|l|l|l|}
\hline error & MAE $\left(^{\circ}\right)$ & MAPE $(\%)$ \\
\hline Elevation & 0.0702 & 0.2095 \\
\hline azimuth & 0.0081 & 0.0023 \\
\hline
\end{tabular}

From the results obtained from the estimation of the error. The heliostat movement does not exceed $0.1^{\circ}$.

\section{CONCLUSION}

We have proposed an estimation model based on recurrent neural networks trained with the backpropagation learning algorithm Levenberg-Marquardt to predict the movements of heliostat fields of a solar tower power plant. This method has explored a new neural network solution based on the supervision and control of the alignment of the heliostat field of a solar power tower.

In this paper, data movement heliostats were simulated based on a procedure using astronomical formulas for the sun's position and characteristics of the plant. The estimation results showed that this model provides an accurate prediction of the movements of heliostats and under the control of the computer. This is an excellent method of estimation for the monitoring and control of the heliostat field of a solar power tower.

\section{REFERENCE}

[1] Kolb,G.J.,Jones,S.A.,Donnelly,M.W.,etal.,2007.Heliostat costreductionstudy,SAND20073293.Availablefrom: $<$ http ://www.prod.sandia.gov/cgibin/techlib/accesscontrol.pl/2 007/073293.pdf>.

[2] William, B.S., Micheal, G., 2001. Power from the sun. Available from: <http://www.powerfromthesun.net/book.htm>.

[3] F M F SIALA, M E ELAYEB, "Mathematical formulation of a graphical method for a no-blocking heliostat field layout," Renewable Energy, 2001, 23(1):77-92.

[4] F. J. COLLADO and J. A. TUREGANO, "Calculation of the annual thermal energy supplied by a defined heliostat field," Solar Energy, 1989, 42(2):149-165.

[5] M. SCHMITZ, P. SCHWARZBO, BUCK R, et al, "Assessment of the potential improvement due to multiple apertures in central receiver systems with secondary concentrators," Solar Energy, 2006, 80(1): 111-120.

[6] F. W. LIPPS, L. L. VANT-HULL, "A cellwise method for the optimization of large central receiver systems," Solar Energy, 1978, 20(6):505-516.

[7] A. KRIBUS, R. ZAIBEL, D. CAREY, et al, "A SOLARDRIVEN COMBINED CYCLE POWER PLANT," Solar Energy, 1998, 62(2):121-129.

[8] Xiudong WEI, Zhenwu LU , Zi LIN , Hongxin ZHANG, Zhengguo NI, " Optimization Procedure for Design of Heliostat Field Layout of a 1MWe Solar Tower Thermal Power Plant, SPIE Vol. 6841 684119-2, 2007.

[9] Ya-Ling He , Fu-Qing Cui, Ze-Dong Cheng, Zeng-Yao $\mathrm{Li}$, Wen-Quan Tao, "Numerical simulation of solar radiation transmission process for the solar tower power plan', Applied Thermal Engineering, 61 (2013) 583595.

[10] Solangi KH, Islam MR, Saidura R, Rahimb NA, Fayaz H. A review on global solar energy policy. Renewable and Sustainable Energy Reviews 2011;15:2149-63.

[11] Kadir Mohd Zainal Abidin Ab, Rafeeu Yaaseen, Adam Nor Mariah. Prospective scenarios for the full solar energy development in Malaysia. Renewable and Sustainable Energy Reviews 2010;14:3023-31.

[12] Chen YT, Kribus A, Lim BH, Lim CS, Chong KK, Karni $\mathrm{J}$, et al. Comparison of two sun tracking methods in the application of a heliostat field. Journal of Solar Energy Engineering 2004;126(1):638-44.

[13] Nian Zhang, Pradeep K. Behera, "solar radiation prediction based on recurrent neural networks trained by levenberg-marquardt backpropagation learning algorithm," IEEE 2011 .

[14] M.T. Hagan and M. Menhaj, "Training Feed-Forward Networks with the Marquardt Algorithm," IEEE Transactions on Neural Networks, vol. 5, no. 6, pp. 989993, 1994. 